\title{
Equoterapia: intervenções terapêuticas e educativas com pessoas com deficiência e com Transtorno do Espectro Autista
}

Hippotherapy: therapeutic and educational interventions with people with disabilities and with Autistic Spectrum Disorder

Hipoterapia: intervenciones terapéuticas y educativas para personas con discapacidad y personas con Trastorno del Espectro Autista

Recebido: 03/10/2021 | Revisado: 11/10/2021 | Aceito: 15/10/2021 | Publicado: 18/10/2021

Gisele Baggio

ORCID: https://orcid.org/0000-0001-8269-3960 Universidade de Passo Fundo, Brasil E-mail:169892@upf.br Karla Duani Bolfe

ORCID: https://orcid.org/0000-0003-1286-1150 Universidade de Passo Fundo, Brasil E-mail: 153828@upf.br

Nicole Werkhausen

ORCID: https://orcid.org/0000-0003-2099-2118 Universidade de Passo Fundo, Brasil E-mail: 143792@upf.br Paulo Cezar Mello

ORCID: https://orcid.org/0000-0003-1749-1187 Universidade de Passo Fundo, Brasil E-mail: pcmello@upf.br

\begin{abstract}
Resumo
Objetiva-se relatar as intervenções terapêuticas e educativas da terapia assistida por cavalos em pessoas com deficiência e com Transtorno do Espectro Autistas (TEA), durante a participação no projeto extensionista em Equoterapia da Universidade de Passo Fundo (UPF). Trata-se de um estudo descritivo, qualitativo, do tipo relato de experiência. Optouse por elencar em tópicos a explanação da vivência, sendo eles: a) quanto a equipe que desenvolve o projeto; b) local e cronograma das atividades; c) quanto os pacientes participantes; d) quanto às intervenções realizadas durante a terapia; e) quanto às intervenções realizadas durante a pandemia. Relataremos as experiências vivenciadas nas sessões de equoterapia englobando características básicas e estruturais do projeto e as intervenções e ações realizadas durante o mesmo. Conclui-se que a vivência com equoterapia proporciona a criação de um elo entre os praticantes e a natureza, fornece resultados físicos, psicológicos, morais, sociais e espirituais para os praticantes e a equipe que a implementa. Promove também uma formação diferenciada, desenvolvendo um trabalho em equipe interdisciplinar e assim, proporcionando uma assistência holística.
\end{abstract}

Palavras-chave: Terapia assistida por cavalos; Terapias complementares; Pessoas com deficiência; Transtorno do Espectro Autista; Equipe de assistência ao paciente.

\begin{abstract}
The objective is to report the therapeutic and educational interventions of horse-assisted therapy in people with disabilities and with Autistic Spectrum Disorder (ASD), during participation in the extension project in Hippotherapy at the University of Passo Fundo (UPF). This is a descriptive, qualitative study, of the experience report type. It was decided to list in topics the explanation of the experience, namely: a) the team that develops the project; b) location and schedule of activities; c) how much the participating patients; d) regarding the interventions performed during therapy; e) regarding the interventions carried out during the pandemic. We will report the experiences lived in the hippotherapy sessions encompassing basic and structural characteristics of the project and the interventions and actions carried out during it. It is concluded that the experience with hippotherapy provides the creation of a link between the practitioners and nature, provides physical, psychological, moral, social and spiritual results for the practitioners and the team that implements it. It also promotes differentiated training, developing interdisciplinary teamwork and thus providing holistic assistance.
\end{abstract}

Keywords: Equine-assisted therapy; Complementary therapies; Disabled people; Autistic Spectrum Disorder; Patient care team. 


\section{Resumen}

El objetivo es dar a conocer las intervenciones terapéuticas y educativas de la terapia asistida por caballos en personas con discapacidad y con Trastorno del Espectro Autista (TEA), durante la participación en el proyecto de extensión en Hipoterapia de la Universidad de Passo Fundo (UPF). Se trata de un estudio descriptivo, cualitativo, del tipo relato de experiencia. Se decidió enumerar en temas la explicación de la experiencia, a saber: a) el equipo que desarrolla el proyecto; b) ubicación y cronograma de actividades; c) cuánto los pacientes participantes; d) con respecto a las intervenciones realizadas durante la terapia; e) sobre las intervenciones realizadas durante la pandemia. Informaremos de las experiencias vividas en las sesiones de hipoterapia englobando las características básicas y estructurales del proyecto y las intervenciones y acciones realizadas durante el mismo. Se concluye que la experiencia con la hipoterapia proporciona la creación de un vínculo entre los practicantes y la naturaleza, brinda resultados físicos, psicológicos, morales, sociales y espirituales para los practicantes y el equipo que la implementa. También promueve la formación diferenciada, desarrollando el trabajo en equipo interdisciplinario y brindando así una asistencia integral.

Palabras clave: Terapía asistida por caballos; Terapias complementarias; Personas con discapacidad; Trastorno del Espectro Autista; Grupo de atención al paciente.

\section{Introdução}

A equoterapia é uma técnica que se utiliza do cavalo como instrumento para possibilitar efeitos terapêuticos e educacionais, com uma abordagem multidisciplinar e associado a um ambiente multissensorial, proporcionando assim, estímulos sensório-motores e buscando o desenvolvimento biopsicossocial de pessoas portadoras ou não de necessidades especiais (Ande, 2021).

O projeto de Lei n. 13.830 de 13 de maio de 2019, regulamenta a prática de equoterapia como uma ferramenta utilizada no tratamento e reabilitação nas áreas de saúde, educação e equitação, considerando que a equipe responsável pela mesma seja multiprofissional (Brasil, 2019).

A semelhança entre o caminhar humano e o do cavalo é demonstrada nos movimentos realizados por ambos, que ocorrem tridimensionalmente, ocupando o plano o ântero posterior, médio lateral e o sagital. Essa característica comum tem sido utilizada na reabilitação, com o objetivo de melhorar padrões de movimento, marcha, força e equilíbrio. Além disso, durante o trajeto da equoterapia é trabalhado a interação social, autoestima, segurança, afetividade, a psicomotricidade, articulações de fala, disciplina, atividades de ensino-aprendizagem, raciocínio lógico e matemático, motoras, sensoriais, entre outras competências e habilidades (Fiuza, 2016).

Destarte, considera-se a equoterapia como um campo novo no âmbito da saúde e educação, e que vem contribuindo para a melhora da qualidade de vida de crianças e adultos sem ou com limitações físicas e psíquicas.

Diante do exposto, objetiva-se relatar as intervenções terapêuticas e educativas da terapia assistida por cavalos em pessoas com deficiência e com Transtorno do Espectro Autistas (TEA), durante a participação no projeto extensionista em Equoterapia da Universidade de Passo Fundo (UPF).

\section{Metodologia}

Trata-se de um estudo descritivo, qualitativo, do tipo relato de experiência acerca da Equoterapia utilizando-a como um método terapêutico e educacional envolvendo pessoas com deficiência.

Define-se relato de experiência como um método de pesquisa descritiva que reflete uma ou mais ações relacionadas e direcionadas à experiência vivida no campo acadêmico de relevância para a comunidade científica (Calado et al., 2019).

Desse modo, para melhor explanação da experiência apresentaremos cada âmbito em forma de tópicos: a) quanto a equipe que desenvolve o projeto; b) local e cronograma das atividades; c) quanto os pacientes participantes; d) quanto às intervenções realizadas durante a terapia; e) quanto às intervenções realizadas durante a pandemia. 


\section{Resultados e Discussões}

a) quanto a equipe que desenvolve o projeto;

Para a execução das sessões de equoterapia, participam discentes de diversos cursos da área da saúde, entre eles, fisioterapia, enfermagem, psicologia, medicina, fonoaudiologia e educação física.

A equipe é composta por 6 alunos bolsistas estagiários paidex, em torno de 20 acadêmicos selecionados por meio de edital e adolescentes que cumprem medida socioeducativa no Centro de Atendimento Socioeducativo (CASE), ambos voluntários. Imprescindível destacar que todos têm suas atribuições e responsabilidades a serem desenvolvidas.

Os bolsistas têm incumbência de propor, criar, planejar e desempenhar atividades para almejar o objetivo do projeto. Os voluntários atuam como terapeutas laterais, acompanhando o paciente e dando o suporte necessário em relação a segurança nos atendimentos. Os adolescentes da CASE ficam responsáveis pelo guiamento do cavalo durante a terapia, além de ajudarem em demandas gerais para a estrutura física do projeto (colocação da rampa).

Ao participar do projeto observamos e percebemos a importância da realização de um trabalho interprofissional e multidisciplinar. Este trabalho por sua vez, em equipe exige muito mais que compartilhar o mesmo espaço, o mesmo consiste na articulação dos diferentes saberes e das práticas profissionais. É essa permanente interação que nos aproxima de um modelo de atenção à saúde mais integral aos pacientes assistidos na terapia (Reeves et al., 2018).

Dessa forma, cabe aos profissionais, sempre de forma prazerosa e lúdica, tendo o cavalo como seu instrumento de trabalho, aplicarem os seus conhecimentos e interagirem trocando informações com os profissionais da equipe, com o intuito de beneficiar o praticante da equoterapia, servindo de elo entre os praticantes e suas famílias e a equipe multiprofissional.

b) local e cronograma das atividades;

A terapia é realizada na Fazenda do $3^{\circ}$ Regimento de Cavalaria Montada da Brigada Militar ( $3^{\circ}$ RPMON/BM), em Passo Fundo, norte do Rio Grande do Sul. Executada todas as segundas feiras e/ou quartas feiras, no período vespertino, das 14:00 às 17:00 horas.

A equoterapia é um método terapêutico diferenciado das consideradas pelo modelo hegemônico, pois é realizada em meio ao ambiente natural, ao ar livre, permitindo assim um maior contato com o meio externo.

A natureza é como um espelho no qual nos vemos, e interagir conectando-se a ela é uma forma de nos integrar com a nossa própria totalidade. Dessa forma, infere-se que a natureza também pode ser tratada como uma ferramenta terapêutica. Assim, a mesma acontece em lugar mais afastado da área central da cidade para que se desfrute da tranquilidade e da natureza que o lugar oferece (Duarte, 2017).

c) quanto os pacientes do projeto;

O projeto atende cerca de 32 pessoas com algum tipo de deficiência. A idade do público atendido engloba partir de 05 até 45 anos de idade, que apresentam diagnóstico de Transtorno do Espectro Autista (TEA), paralisia cerebral, traumatismo neurológico, hidrocefalia, síndromes de rett e west, distúrbios neuromotores e osteomusculares.

c.1) quanto aos alunos com síndromes de rett, síndrome de west, traumatismo neurológico, hidrocefalia, distúrbios neuromotores e osteomusculares;

O sistema nervoso central é um órgão de reação ao invés de ação e reage aos estímulos que para ele convergem a partir de fora e de dentro do corpo. A função mais importante do sistema nervoso central é sua capacidade de inibir atividades descoordenadas ou indesejadas ao mesmo tempo em que promove funções práticas, possibilitando o armazenamento de informações, ou seja, a capacidade de aprender. 
O campo de ação da Equoterapia é bastante amplo e nele as pessoas com deficiências sensório motoras podem participar, tais como: Tipos clínicos de paralisia cerebral, Déficits sensoriais, Atraso maturativo, Síndromes neurológicas (Down, West, Rett, Soto e outras), Acidente Vascular Encefálico, Traumatismo cranioencefálico, Sequelas de processo inflamatórios do sistema nervoso central (meningo-encefalite e encefalite), Lesão raquimedular, entre outras (Medeiros \& Dias, 2002).

Percebe-se que independente da patologia que o participante tem, ao participar da terapia, ele se beneficia a níveis físico, psíquico e social, sendo o cavalo o principal responsável das ações mencionadas anteriormente.

O cavalo se deslocando ao passo transmite ao praticante, uma série de movimentos que resultando no movimento tridimensional (Orsolin et al., 2012), é este aspecto que auxilia e possibilita a melhoria no estado em que se encontra o paciente.

\section{c.2) quanto aos alunos com TEA:}

O Transtorno do Espectro Autista (TEA), era antigamente denominado e conhecido como autismo. Consiste numa síndrome neurocomportamental que é caracterizada por acometer o desenvolvimento cognitivo e social, a linguagem, além da presença e/ou existência de comportamentos repetitivos realizados pela pessoa que apresenta a síndrome. (Mukherjee et al., 2021). Sendo o seu grau/intensidade bastante variado, podendo existir casos muito severos, comprometendo drasticamente a capacidade laboral das pessoas, como há casos considerados leves (Leopoldino, 2015).

Em um estudo que teve como objetivo investigar a contribuição da equoterapia para o desenvolvimento psicomotor de crianças com TEA, obteve-se como resultados e apontamentos que os ganhos trazidos pela Equoterapia para as pessoas com TEA são grandes, relacionando tanto aspectos físicos, quanto mentais e sociais, pois, o contato com o cavalo estimula os movimentos do corpo e por consequente, a movimentação física, e também faz com que a criança crie afeição pelo animal, e como efeito cria interação/vínculo com as pessoas, propiciando assim o desenvolvimento biopsicossocial do praticante (Quinteiro \& Pottker, 2017).

\section{c.3) quanto aos alunos com paralisia cerebral:}

A paralisia cerebral consiste em uma alteração neurológica que desencadeia em desordens motoras e posturais, interferindo nos movimentos e postura das crianças e portadores de tal patologia. Dependendo da localização da região lesada, essas alterações variam, sendo as mais encontradas: a espasticidade, hipotonia, comprometimento cognitivo, sensorial, visual, auditivo e o equilíbrio (Graham et al., 2016 \& Hidecker et al., 2018)

Com estudos evidenciou-se benefícios significativos na função motora grossa (Mutoh et al., 2018) e no equilíbrio (Vargas et al., 2016) em vários níveis funcionais quando o paciente realiza equoterapia.

Ademais, apresentam melhoras na psicomotricidade, controle de tronco e movimentos da cabeça, assim como o desempenho funcional de habilidades da vida diária em pessoas com leve e moderados problemas motores (Hsieh et al., 2017).

Expõe-se aqui alguns estudos sobre TEA e PC, por apresentarem prevalência nos atendimentos da equoterapia. Contudo, infere-se que as pessoas que possuem deficiência e/ou TEA e que participam desta terapia, fazem parte de sua própria reabilitação, por exemplo, praticante desenvolve um relaxamento corporal e mental, fundamental para alcançar a postura almejada e o alinhamento do centro de gravidade, durante o percurso. Já, para montar e apear do cavalo, desenvolve-se uma conscientização do próprio corpo, assim como um aperfeiçoamento da coordenação motora, desenvolvendo sua noção de equilíbrio. E, não menos importante, a própria interação com o cavalo, abarcando os primeiros contatos e aproximações, os cuidados e manejos preliminares, a ação de montar e o manuseio final, desenvolvem, ainda, novas formas de socialização, autoestima e autoconfiança (Silva \& Salles, 2018). 


\section{d) quanto às intervenções realizadas durante o trajeto;}

A cada terapia, o praticante realiza as mesmas tarefas de esperar, montar, sair ao passo, realizar atividades lúdicas e pedagógicas nas ilhas da educação física e fonoaudiologia durante o percurso e apear no final da sessão.

Para a escolha do cavalo a ser utilizado na terapia leva-se em consideração o tamanho, altura e peso do paciente e o comportamento do animal, assim como os materiais de montaria a serem utilizados: estribo e sela.

De início é realizada a aproximação, onde existe a criação do vínculo afetivo do paciente praticante com o animal que será montado. É incentivado que o praticante chame o cavalo, acaricie-o e de forma gradativa, até o mesmo ser colocado sobre o animal. Também, de acordo com a aceitabilidade da atividade antes mesmo dessa etapa acontecer, pode ser realizada uma intervenção lúdica que tem por objetivo demonstrar as etapas aos pacientes do que será realizado durante a sessão por meio de uma planilha com figuras com velcro para que ele possa visualizar e estabelecer sua própria rotina antes do início da sessão

Durante a sessão, o paciente praticante é acompanhado devidamente pela equipe multidisciplinar. Segundo Bueno e Moreira (2019), é correto afirmar que, o trabalho da equipe multidisciplinar visa avaliar o paciente de maneira independente e executando seus planos de tratamento como uma "camada adicional" de serviços [...], o principal aspecto positivo da atuação em equipe interdisciplinar é a possibilidade de colaboração de várias especialidades que denotam conhecimentos e qualificações distintas. Assim, a integração da equipe de saúde é imprescindível para que o atendimento e o cuidado alcancem a amplitude do ser humano, transcendendo a noção de conceito de saúde.

Cada praticante é considerado um ser único, dando-se prioridade para a subjetividade de cada sujeito, dessa maneira as intervenções na terapia ocorrem de acordo com as demandas do praticante, preocupando-se com a ética, respeitando os direitos humanos, o meio ambiente e os cavalos.

Ou seja, quem define a necessidade é o praticante e quem comanda a sessão é o cavalo. A equipe planeja, assiste e acompanha todos os passos, devolvendo ao paciente praticante e ou responsável, seu desempenho e conquistas.

Ao longo da terapia é realizada uma pausa no trajeto com o cavalo e executando atividades nas chamadas ilhas da educação física e fonoaudiologia. $\mathrm{O}$ trabalho em geral precisa ser desenvolvido de forma personalizada e única, não sendo possível fazer comparações de desenvolvimento entre os participantes, mesmo porque, aquele momento é ricamente individual.

$\mathrm{Na}$ ilha da Educação Física são realizadas atividades específicas para cada paciente que promovem o alongamento e estimulação do equilíbrio, postura, força, entre outros. Os exercícios sobre o cavalo são realizados com diferentes técnicas lúdicas e terapêuticas, respeitando os limites de cada um e exigindo de acordo com suas possibilidades para atingir os objetivos planejados.

Os exercícios físicos realizados de forma regular ou frequente estimulam o sistema imunológico e trazem benefícios para saúde tanto física quanto mental. A prática regular ajuda a aumentar os níveis de energia e pode ajudar a prolongar a vida. De acordo com Zuchetto e Castro (2002) a prática de exercícios não só contribui para a melhoria dos aspectos físicos quanto psicológicos das pessoas com necessidades especiais, como também na melhoria das capacidades funcionais, permitindo uma melhor mobilidade.

É indiscutível que a Educação Física com a sua fundamentação no desenvolvimento humano, práticas psicomotoras e pedagógicas podem contribuir no processo de aprendizagem e reabilitação do paciente praticante da Equoterapia.

Segundo Gorgatti e Costa (2005) a Educação Física Adaptada tem bastantes acontecimentos semelhantes a Equoterapia e também faz parte de um conjunto de atividades e exercícios, que permite ao praticante um desenvolvimento individualizado de aptidão física e motora, habilidades e padrões motores fundamentais e habilidades de vários tipos.

Neste sentido, a Equoterapia oferece um enorme campo de atuação e a Educação Física tem um importante papel a desempenhar, participando não somente no desenvolvimento de habilidades, mas, também criando condições fundamentais que objetivem a formação plena do paciente. 
O educador físico pode cooperar para a melhoria das condições físicas dos praticantes, fazendo uma consonância com as ações desenvolvidas com o auxílio do cavalo. Um cavalo em movimento auxilia os praticantes a se familiarizar com mecanismos de equilíbrio e a melhorar reações adversas. E a melhora no equilíbrio proporciona melhor alinhamento postural, tônus muscular, controle de cabeça e tronco, controle das extremidades e da coordenação (Shkedi \& Engel, 1997).

No âmbito da Fonoaudiologia, são realizadas diversas atividades que visam beneficiar o paciente com disfunções neurológicas e do desenvolvimento infantil, com alterações diversas causadas por alguma síndrome, Transtorno do Espectro Autista (TEA), crianças e adultos com distúrbios sensoriais, emocionais, de aprendizagem e comportamentais. Os exercícios são exclusivamente pensados em cada praticante da equoterapia, levando em consideração suas dificuldades e limitações.

O fonoaudiólogo exerce papel muito importante na equipe de equoterapia, além de participar da discussão de casos e fortalecer a integração entre o praticante e o animal, está diretamente relacionado às barreiras da linguagem, fonoaudiológicas (Valle, Nishimori, \& Nemr, 2014). A fonoaudiologia atua diretamente na estimulação sensorial, motricidade orofacial, motricidade ampla e fina, linguagem oral e escrita, leitura, números e cores, além de estar sempre estimulando a comunicação dos praticantes do projeto, seja ela verbal ou não verbal. Apesar da estimulação da comunicação ser realizada durante todo o percurso pelos terapeutas laterais, a ilha da fonoaudiologia também é um momento de interação, onde o espaço é aberto para diálogo entre terapeuta e praticante.

Como parte da equipe, a enfermagem atua principalmente prestando orientações em saúde para o grupo de pais/responsáveis sobre temas pertinentes, realizando atividades de autoconhecimento e ações voltadas à saúde mental dos mesmos. Também cuida do entrosamento do praticante com o animal, assim como na função motora.

Percebe-se a demanda por profissionais da área, tornando-os um elemento-chave no grupo de pais e na prestação de orientações sobre educação em saúde, na prestação dos primeiros socorros, na prevenção de acidentes, bem como na construção de um plano terapêutico singular, contribuindo sob a ótica do cuidado para os praticantes da equoterapia, assim como para seus cuidadores de forma biopsicossocial.

A enfermagem é a essência da profissão e pertence a dois campos distintos: o campo objetivo, que se refere ao desenvolvimento de tecnologias e procedimentos, e o campo subjetivo, que cuida do outro com base na sensibilidade e criatividade para cuidar de outro ser. Ultrapassa o senso comum de uma atenção pontual e episódica para se constituir em uma atenção contínua, sistematizada e contextualizada (Santos, et al., 2017).

Para o encerramento da sessão o praticante é incentivado a despedir-se do animal, acariciando-o e alimentando o animal. Ao término de todas as sessões no dia respectivo, todo o trabalho desenvolvido é registrado em cadernos, que servem como prontuários, onde neles contém: nome do praticante, nome dos integrantes da equipe pelo qual foi atendido, evolução da sessão, recursos utilizados, estado do praticante e intercorrências durante o trajeto. As anotações são discutidas e levantadas a uma roda de conversa com os acadêmicos estagiários participantes do projeto, que promove a participação entre a equipe interprofissional, alinhando demandas, necessidades encontradas e indagações pertinentes, permitindo assim, a continuidade das atividades em sessões futuras.

e) quanto às intervenções realizadas durante a pandemia.

Atualmente, no ano de 2020, o mundo vivencia uma pandemia ocasionada pelo novo coronavírus, o vírus SARS-CoV2, causador da doença denominada COVID-19. É uma doença nova que atinge o sistema respiratório e foi identificada pela primeira vez em Wuhan, na China, em dezembro de 2019 (World Health Organization, 2020). Sua transmissão ocorre a partir da disseminação do vírus de uma pessoa doente para outra, ou por contato próximo por meio do aperto de mão, gotículas de saliva, espirro, tosse, catarro, objetos e/ou superfícies contaminadas, tendo como sintomas a febre, tosse ou dificuldade para respirar, entre outros sintomas gripais (Ministério da Saúde, 2020). 
Para evitar a propagação da doença, a Organização Mundial de Saúde (OMS) declarou no começo do mês de março de 2020 que o surto da Covid-19 evoluiu para uma pandemia mundial. Diante disso, as atividades do Projeto de Extensionista Equoterapia foram suspensas, iniciando um novo processo de execução das atividades extensionistas.

Após reuniões online com os acadêmicos estagiários retomou-se às atividades com vídeo orientando com cartazes explicando para os praticantes da equoterapia a respeito dos cuidados em relação a pandemia da Covid-19, auxiliando-os na compreensão deste novo cenário. Posteriormente, outro vídeo foi realizado, este através de fotos com todas as pessoas com deficiência participantes da equoterapia, relembrando momentos marcantes das atividades de equoterápicas na Fazenda da Brigada Militar.

Nos meses seguintes, o Projeto Equoterapia seguiu com reuniões sistemáticas com a equipe de estagiários, iniciando realizações de lives de forma online, estas foram realizadas mensalmente, no turno da noite para que as pessoas com deficiência pudessem participar juntos com seus familiares. Para a efetivação destas lives foram convidados profissionais da área da saúde, a ideia era seguir dando suporte emocional e social a estas famílias beneficiadas.

Em novembro de 2020 foi possível retornar com as atividades de campo de equoterapia, utilizando-se protocolos de atendimentos e os EPIs obrigatórios (Equipamentos de Proteção Individual), para a efetivação do trabalho de campo, sendo chamado para as atividades de equoterapia um pequeno grupo de beneficiários.

Em maio de 2021, as atividades continuaram seguindo todas as medidas de cuidado e prevenção contra a COVID-19, seguindo autorização dos órgãos reguladores. Atualmente, estamos atuando nas segundas-feiras pela parte da tarde, atendendo pacientes que não necessitam tanto de contato como uma montaria dupla. Para dar início a sessão o paciente deve estar utilizando máscara facial, capacete e deve colocar o protetor face shield, além de higienizar as mãos, assim como, os que o acompanham devem estar de máscara. Durante o trajeto, antes e após realizar as atividades nas ilhas, todos os materiais utilizados são higienizados, assim como no término das atividades do dia, sendo que essas medidas visam a continuidade das atividades do projeto e a manutenção da saúde.

Pode-se evidenciar por meio da observação que todo o processo de equoterapia, juntamente com as atividades realizadas nas ilhas, têm eficiência e impacto positivo, tanto para os praticantes como para seus familiares. O que parece um simples passeio de cavalo, na verdade, é um tratamento considerado uma prática integrativa e complementar, sendo que os praticantes cavalgam rumo a uma melhor qualidade de vida e saúde.

Tal assertiva mencionada anteriormente vai de encontro ao descrito no relato de experiência, feito por Bueno \& Monteiro (2010), onde a prática apontou significativa melhora no bem-estar e saúde dos participantes. Também, seguindo nesta linha de pensamento, uma revisão sistemática realizada demonstrou que os benefícios abrangem os praticantes de diferentes faixas etárias, classes sociais e condições de saúde (Madrá, et al., 2019).

\section{Considerações Finais}

A vivência com equoterapia proporciona a criação de um elo entre os praticantes e a natureza, fornece resultados físicos, psicológicos, morais, sociais e espirituais para os praticantes e a equipe que a implementa. Promove também uma formação diferenciada, desenvolvendo um trabalho em equipe interdisciplinar e assim, proporcionando uma assistência holística.

Assim, verificou-se que esta prática: evidenciou melhoras nas relações dos familiares/cuidadores, pois os praticantes melhoram em aspectos físicos e psíquicos e os pais, por sua vez, puderam ter um espaço para falar de suas vivências e experiências, criando uma rede de apoio; proporcionou aos acadêmicos experiências novas, integração entre teoria e prática, aprendizagens e troca de conhecimento entre as pessoas da equipe; e atendeu as demandas do paciente como um todo.

Portanto, devido à importância deste tema, recomenda-se a realização de mais pesquisas sobre o assunto, culminando em contribuições em nível científico e social. 


\section{Referências}

Ande (2021). O método equoterapia. Ande-Associação Nacional de Equoterapia. Brasil.http://equoterapia.org.br/articles/index/article_detail/142/2022.

Brasil. (2019). Lei n 13.830, de 13 de maio de 2019 que dispõe sobre a prática da equoterapia. https://legis.senado.leg.br/norma/3 0940789/publicacao/30940896

Bueno, M. B. T. \& Moreira, M. I. G. (2019). Estudo da cooperação na equipe multidisciplinar da modalidade de equoterapia, na Associação de Pais e Amigos dos Excepcionais, no município de Pelotas/RS. Revista de Gestão e Organizações Cooperativas., 5(10):125. https://www.researchgate.net/publication/333692092_Estudo_da_cooperacao_na_equipe_multidisciplinar_da_modalidade_de_equoterapia_na_Associacao_de _Pais_e_Amigos_dos_Excepcionais_no_municipio_de_PelotasR

Bueno, R. K. \& Monteiro, A. M. (2010). Estudante de Psicologia na Equoterapia: Um relato de experiência. Revista Eletrônica de Extensão da URI. https://www.researchgate.net/publication/262728858_Estudante_de_Psicologia_na_Equoterapia_Um_relato_de_experiencia

Fiuza, J. (2016). Equoterapia como recurso pedagógico: dificuldades de aprendizagem. Dissertação de Mestrado em Práticas Socioculturais e Desenvolvimento Social do Programa de Pós-Graduação Stricto Sensu da Universidade de Cruz Alta - UNICRU. https://home.unicruz.edu.br/wpcontent/uploads/2017/03/Jaquelini-Fiuza-EQUOTERAPIA-COMO-RECURSO-PEDAGOGICO-DIFICULDADES-DE-APRENDIZAGEM.pdf

Calado, R. S. F., Silva, A. A. O. B., Oliveira, D. A. L., Silva, G. A. M., Silva, J. C. B., Silva, L. C., Lemos, M. E. P. \& Santos, R. C. (2019). Ensino das práticas integrativas e complementares na formação em enfermagem. Revista de Enfermagem UFPE., 13(1): 261-267. https://pesquisa.bvsalud.org/portal/resource/pt/biblio-1007745

Duarte, A. J. O. (2017). Ecologia da alma: a natureza na obra científica de Carl Gustav Jung. Junguiana., 35(1):05-19. http://pepsic.bvsalud.org/scielo.php?script=sci_arttext\&pid=S0103

$-08252017000100002 \& \operatorname{lng}=$ pt\&nrm=iso.

Gorgatti, M.G. \& Costa, R.F. (2005). Atividade física adaptada. https://www.efdeportes.com/efd210/educador-fisico-na-equoterapia.htm.

Graham, H. K., Rosenbaum, P., Paneth, N., Dan, B., Lin, J. P., Damiano, D. L., Becher, J. G., Gaebler-Spira, D., Colver, A., Reddihough D. S., Crompton, K. E. \& Lieber, R. L. et al. (2016). Cerebral palsy. Nat Rev Dis Primers. 2:15082

Hidecker, M. J. C., Slaughter, J., Abeysekara, P., Ho. N. T., \& Dodge, N., Hurvitz, E. A., Workinger, M. S., Kent, R. D., Rosenbaum, P., Lenski, M., Vanderbeek, S. B., DeRoos, S. \& Paneth, N. (2018). Early Predictors and Correlates of Communication Function in Children with Cerebral Palsy. Journal of Child Neurology, 33(4): 255-59. https://doi.org/10.1177/0883073817754006.

Hsieh, Y. L., Yang, C. C, Sun, S. H, Chan, S. Y, Wang, T. H, \& Luo, H. J. (2017). Effects of hippotherapy on body functions, activities and participation in children with cerebral palsy based on ICF-CY assessments. Disability and Rehabilitation., 39 (17):1703-1713. http://dx.doi.org/10.1080/09638288.2016.1207108

Leopoldino, C. B. (2015). Inclusão de autistas no mercado de trabalho: uma nova questão de pesquisa. Revista Eletrônica Gestão \& Sociedade, 9(22):853-868. https://www.gestaoesociedade.org/gestaoesociedade/article/view/2033

Mandrá, P. P., Moretti, C. F., Avezum, L. A. \& Kuroishi, R. C. S. (2019). Terapia assistida por animais: revisão sistemática da literatura. CoDAS. 31(3). https://doi.org/10.1590/2317-1782/20182018243

Medeiros, M., Dias, E. (2002). Equoterapia: bases e fundamentos. Revinter.

Mukherjee, S. B., Kapoor, S. \& Sharma, S. (2021). Identification of Essential, Equivocal and Complex Autism by the Autism Dysmorphology Measure: An Observational Study. Journal of Autism and Developmental Disorders, 51(5), 1550-1561, https://doi.org/10.1007/s10803-020-04641-X

Ministério da Saúde. (2020). Coronavírus e novo coronavírus: o que é, causas, sintomas, tratamento e prevenção. https://www.saude.gov.br/saude-de-az/coronavirus.

Mutoh, T., Mutoh, T., Tsubone, H., Takada, M., Doumura, M., \& Ihara M., et al. (2018). Impact of serial gait analyses on long-term outcome of hippotherapy in children and adolescents with cerebral palsy. Complement Ther Clin Pract., 30: 19-23. http://dx.doi.org/10.1016/j.ctcp.2017.11.003

Orsolin, T. O. P. Neto, V. E. P., Santos, P. L. S. Saes, M. O. (2012). A influência da variação do peso na frequência do passo do cavalo. Ensaios e Ciência: Ciências Biológicas, Agrárias e da Saúde, 16(3),39-48. https://www.redalyc.org/articulo.oa?id=26029237004

Quinteiro, B. C., \& Pottker, C. (2017). As contribuições da Equoterapia para o desenvolvimento psicomotor da criança com tran storno de espectro autista. Revista Uningá Review, 32(1):147-158. http://revista.uninga.br/index.php/uningareviews/article/view/143

Reeves S., Xyrichis A., \& Zwarenstein M. (2018). Teamwork, collaboration, coordination, and networking: why we need to distinguish between different types of interprofessional practice. Journal of Interprofessional Care., 32(1):1-3. https://www.tandfonline .com/doi/full/10.1080/13561820.2017.1400150

Santos, A. G., Monteiro, C. F. M., Nunes, B. M. V. T., Benício, C. D. A. V., \& Nogueira, L. T. (2017). O cuidado em enfermagem analisado segundo a essência do cuidado de Martin Heidegger. Revista Cubana de Enfermería, 33(3). http://www.revenfermeria.sld.cu/index.php/enf/article/view/1529/295

Shkedi, A., \& Engel, B. T. (1997). Emprego da equoterapia no tratamento das disfunções do desenvolvimento neurológico. Proceeding of the 9th International Therapeutic Riding Congress. https://www.efdeportes.com/efd210/educador-fisico-na-equoterapia.htm.

Silva, A. S. M., Lima, F. P. S., \& Salles, R. J. (2018). Vínculo afetivo de crianças autistas na equoterapia: uma contribuição de Winnicott. Boletim - Academia Paulista de Psicologia, 38(95), 238-250. http://pepsic.bvsalud.org/scielo.php?script=sci_arttext\&pid=S1415-711X2018000200011\&lng=pt\&tlng=pt.

Zuchetto, A. T., \& Castro, R. L. V. G. (2002). As contribuições das atividades físicas para a qualidade de vida dos deficientes físicos. Revista Kinesis., 26:52166. https://periodicos.ufsm.br/kinesis/article/view/8010/4793 
Research, Society and Development, v. 10, n. 13, e438101321353, 2021

(CC BY 4.0) | ISSN 2525-3409 | DOI: http://dx.doi.org/10.33448/rsd-v10i13.21353

Valle, L. M. O., Nishimori, A. Y., \& Nemr, K. (2014). Atuação fonoaudiológica na equoterapia. Revista CEFAC., 16(2):511-523. https://doi.org/10.1590/19820216201420712

Vargas L. P. J, Cartas U. S, Larrarte J. P. M, \& Espinosa I. S. (2016). Aplicación de la hipoterapia en los niños con parálisis cerebral. Rev. Arch Med Camagüey. 20(5): 496-506. http://scielo.sld.cu/pdf/amc/v20n5/amc060516.pdf.

World Health Organization. (2020). IHR procedures concerning public health emergencies of international concern (PHEIC), http://www.who.int/ihr/procedures/pheic/en/ 